\title{
The Warburg Effect - An Onco-hematologic Emergency?
}

\author{
Poggi Guido*, Montagna Benedetta and Gordon Shaul \\ UO Oncologia Istituto Città di Pavia, Italy \\ Facoltà di Medicina e Chirurgia, Università degli studi di Pavia, Italy
}

Submission: April 06, 2021; Published: April 21, 2021

*Corresponding author: Prof Guido Poggi, UO Oncologia Istituto Città di Pavia, via Parco Vecchio 27 Pavia, Italy

\begin{abstract}
Warburg effect is a rare and potentially life-threatening metabolic complication occurring in oncologic patients. The Authors report the case of a 79-year-old man affected by diffuse large B-cell lymphoma presenting with severe lactic acidosis and hypoglycaemia both of which were refractory to conventional management and they critically review the available literature.

Keywords: Warburg effect; Lactic acidosis; Hypoglycaemia; Hematologic malignancies; Cancer cells; Chronic hepatitis C; Nephrology; Bladder tumour
\end{abstract}

\section{Introduction}

The combination of lactatemia and severe untreatable hypoglycaemia is a rare metabolic complication of hematologic malignancies. It occurs more frequently in patients affected by lymphoma, and it is generally associated with an ominous prognosis. It is secondary to an avid consumption of glucose by the cancer cells that even under aerobic conditions switch their glucose metabolism from the oxidative pathway to the glycolytic pathway, leading to increased lactate production. This metabolic shift is known as the "Warburg effect". Here we will discuss a patient with diffuse large B-cell lymphoma (DLBCL), who presented with hypoglycaemia and lactic acidosis, and we will review similar cases from the relevant literature, which we believe warrant the consideration of the Warburg effect as an onco-hematological emergency.

\section{Case Description}

A 79-year-old man was referred to our ward for evaluation of massive hepatomegaly discovered two weeks earlier, when he presented to the emergency department complaining of weight loss, nausea, vomiting and abdominal discomfort. In the emergency department, he was diagnosed with an acute renal injury of prerenal origin, presumptive subacute cholangitis and chronic hepatitis C. He was transferred for evaluation in the nephrology department and received treatment with fluid replacement, resulting in partial improvement of renal function. He was subsequently discharged and referred to us to complete the diagnostic workup. At the time of admission, his therapeutic regimen included edoxaban and flecainide, as well as amlodipine and losartan for arterial hypertension. His medical history was notable for tuberculous spondylodiscitis at age 20 , and a cholecystectomy at age 46. In 2017, two years before he was referred to us, he was diagnosed with transitional cell bladder carcinoma that did not infiltrate the muscular layer and was treated with transurethral resection of a bladder tumour (TURBT) followed by intravesical chemotherapy. He was also diagnosed with atrial fibrillation and treated with direct-acting oral anticoagulants (DOAC), and in 2018 he required electric cardioversion. Familial history was notable for the untimely death of his mother at age 35 for a hepatic tumour, and the death of his brother at the age of 66 , for multiple myeloma.

Upon arriving at our ward, the patient was alert and presenting no noticeable neurological alterations. He appeared unfit, complained of profound fatigue, anorexia and abdominal discomfort. He reported to have lost approximately $10 \mathrm{~kg}$ in the previous two months and denied any episodes of fever or drenching sweats. His temperature was $36.8{ }^{\circ} \mathrm{C}$, the heart rate 


\section{Juniper Online Journal of Case Studies}

was 98 beats per minute, the respiration rate was 18 breaths per minute, and the oxygen saturation was at $97 \%$ while breathing ambient air. Physical examination revealed massive hepatomegaly, extending on the lower margin to $2 \mathrm{~cm}$ below the transverse umbilical line, and concomitant splenomegaly with a palpable lower pole $4 \mathrm{~cm}$ below the costal arch. The ultrasound confirmed massive hepatosplenomegaly; however, the hepatic parenchyma revealed no nodules, but only a focal solitary hypoechoic area of non-nodular appearance. A contrast-enhanced total body CT scan was performed and confirmed the ultrasound findings with liver and splenic homogeneous enlargement and further revealed the involvement of multiple retroperitoneal lymphnodes not found at the US scan. A complete blood count, including a metabolic panel and arterial blood gas analysis, was performed (Table 1-2), revealing significant hypoglycaemia and metabolic acidosis with a high anion gap, consistent with lactic acidosis.

Table 1: Values from the blood count and metabolic panel.

\begin{tabular}{|c|c|c|c|}
\hline Variable & Unit of Measurement & Result & Normal Range \\
\hline BUN & $\mathrm{mg} / \mathrm{dl}$ & 138 & $10-50$ \\
\hline Creatinine & $\mathrm{mg} / \mathrm{dl}$ & 2.12 & $0.7-1.2$ \\
\hline Uricemia & $\mathrm{mg} / \mathrm{dl}$ & 11.4 & $3.4-7.0$ \\
\hline Bilirubin & $\mathrm{mg} / \mathrm{dl}$ & 5.2 & $<1.2$ \\
\hline Glucose & $\mathrm{mg} / \mathrm{dl}$ & 28 & $14-110$ \\
\hline Hemoglobin & $\mathrm{g} / \mathrm{dl}$ & 13.5 & $150-450$ \\
\hline Platelets & $\mathrm{X} 10^{3} / \mathrm{dl}$ & 277 & $240-480$ \\
\hline LDH & $\mathrm{mg} / \mathrm{dl}$ & 139 & $<41$ \\
\hline ALT & $\mathrm{IU} / \mathrm{l}$ & 85000 & Negative \\
\hline HCV-RNA viremia & $\mathrm{IU} / \mathrm{ml}$ & & \\
\hline
\end{tabular}

Table 2: Blood gas analysis.

\begin{tabular}{|c|c|c|}
\hline Variable & Unit of Measurement & Result \\
\hline $\mathrm{pH}$ & - & 7.25 \\
\hline $\mathrm{pCO}_{2}$ & $\mathrm{~mm} / \mathrm{Hg}$ & 29 \\
\hline $\mathrm{pO}_{2}$ & $\mathrm{~mm} / \mathrm{Hg}$ & 12.3 \\
\hline $\mathrm{HCO}-$ & $\mathrm{mmol} / \mathrm{l}$ & 139 \\
\hline $\mathrm{Na}+$ & $\mathrm{mmol} / \mathrm{l}$ & 4.5 \\
\hline $\mathrm{K}+$ & $\mathrm{mmol} / \mathrm{l}$ & 100 \\
\hline $\mathrm{Cl}$ & $\mathrm{mmol} / \mathrm{l}$ & 3.1 \\
\hline Albumin & $\mathrm{mg} / \mathrm{dl}$ & 16.7 \\
\hline Lactate & $\mathrm{mmol} / \mathrm{l}$ & 20,3 \\
\hline Anion gap & $\mathrm{mmol} / \mathrm{l}$ & \\
\hline
\end{tabular}

Despite the severe hypoglycaemia, the patient was not complaining of neuroglycopenic symptoms. Endocrinological investigation showed that the adrenal and thyroid functions were normal. Other markers, including insulin, pro-insulin and C-peptide, were all reduced, excluding both endogenous insulin production and exogenous administration as a cause for hypoglycaemia. Biopsies of the bone marrow and liver revealed a massive infiltration of diffuse large B-cell lymphoma (DLBCL), expressing CD 20+, CD 30+ and EBV+ with high proliferative activity (Ki-67 $=90 \%)$. Correction of the hypoglycaemia with a continuous IV infusion of a $10 \%$ glucose solution and thiamine failed and exacerbated the lactatemia. Therefore, since the patient did not present hypoglycaemic symptoms, the attempt to correct the hypoglycaemia was discontinued. Unfortunately, the rapid progression of thrombocytopenia and the progressive increase in creatinine levels excluded chemotherapy as a therapeutic option. The patient, therefore, received treatment with prednisone $(60 \mathrm{mg} /$ day $)$ and a weekly regimen of rituximab, which led to a gradual improvement of both the clinical presentation and the blood parameters over the first three administrations of rituximab. However, shortly after that, the clinical picture rapidly worsened, and the patient died.

\section{Discussion}

The clinical presentation of Non-Hodgkin's Lymphoma (NHL) varies depending on the subtype and the involved sites, with symptoms including enlarged palpable lymphadenopathy, B-symptoms (fever, weight loss, night sweats), and symptoms 
secondary to compression of adjacent structures. In rare cases, the clinical presentation may include metabolic complications [1-3] such as lactic acidosis and hypoglycemia. Lactate is a by-product of glucose metabolism, under anaerobic conditions, when pyruvate is reduced to lactate by lactate dehydrogenase ( $\mathrm{LDH}$ ). In normal conditions, most of the lactic acid is cleared by the liver (8090\%) and converted to glucose through gluconeogenesis (Cori's cycle), while the remainder is secreted by the kidneys [4]. Lactate accumulation leads to lactic acidosis when the concentration of lactate in whole blood exceeds $5 \mathrm{mmol} / \mathrm{L}$ with a pH below 7.35 [3]. Lactic acidosis can result either from hypoperfusion (type A) or from overproduction or decreased clearance of lactate (type B). Type A lactic acidosis can result from clinical settings of hypoxia and inadequate tissue perfusion due to septic or hypovolemic shock. Type B, however, occurs in normal perfusion states and is associated with malignancies, underlying liver or kidney failure, diabetes mellitus, thiamine deficiency, drugs and toxins (e.g., alcohols, metformin, salicylates, reverse transcriptase inhibitors, cyanides) and hereditary enzymatic defects $[5,6]$. In the case of malignancies, as observed by Otto Warburg many years ago, cancer cells consume glucose and excrete lactate at a significantly higher rate compared to healthy cells, even in normoxic conditions [7]. The increased rate of glycolysis is due to the aberrant expression or over-expression of glycolytic enzymes, as part of the malignant process. One such example is hexokinase II, a rate-limiting enzyme involved in glycolysis, whose activity is regulated by the IGF signalling pathway, which is often defective in malignancies $[8,9]$. However, even though cancer cells produce increased amounts of lactate, lactic acidosis does not develop until the measure of lactate exceeds the limit of hepatic clearance and overwhelms the renal clearance, which could happen when the underlying disease has developed multiple metastases or diffused infiltration of the liver [10], as it is in the majority of reported cases of leukaemia and lymphoma and in the case we report here.

Table 3: Literaure Review.

\begin{tabular}{|c|c|c|c|c|c|c|c|c|c|c|c|c|c|}
\hline Case & Age & Sex & Diagnosis & $\begin{array}{l}\text { Hypo- } \\
\text { glyce- } \\
\text { mia } \\
\text { (mg/ } \\
\text { dl) }\end{array}$ & pH & $\begin{array}{c}\text { Serum } \\
\text { Lactate } \\
(\mathrm{mmol} / \mathrm{L})\end{array}$ & $\begin{array}{c}\text { Liver } \\
\text { Involve- } \\
\text { ment }\end{array}$ & $\begin{array}{l}\text { Serum } \\
\text { Glucose } \\
\text { (mg/ } \\
\text { dl) }\end{array}$ & Therapy & $\begin{array}{l}\text { Out- } \\
\text { come }\end{array}$ & $\begin{array}{c}\text { Survival } \\
\text { (Months) }\end{array}$ & Year & Ref \\
\hline 1 & $64 y$ & $\mathrm{~F}$ & DLBCL & Y & 7.24 & 28.5 & Y & 26 & RCHOP & Fatal & 1 & 2012 & 15 \\
\hline 2 & $55 y$ & F & DLBCL & $\begin{array}{l}\text { Nor- } \\
\text { mal }\end{array}$ & 7.17 & 12.7 & $\mathrm{~N}$ & Normal & $\begin{array}{c}\text { CHOP + } \\
\text { intrathecal } \\
\text { MTX }\end{array}$ & Fatal & 1 & 2011 & 16 \\
\hline 3 & $45 y$ & M & $\begin{array}{l}\text { Primary Os- } \\
\text { seous NHL }\end{array}$ & Y & $\begin{array}{l}\text { Lactic } \\
\text { acido- } \\
\text { sis }\end{array}$ & 16.05 & Y & NR & $\begin{array}{c}\text { Chemother- } \\
\text { apy }\end{array}$ & $\begin{array}{l}\text { Favor- } \\
\text { able }\end{array}$ & NR & 2010 & 17 \\
\hline 4 & $53 y$ & M & DLBCL & Y & $\begin{array}{l}\text { Lactic } \\
\text { acido- } \\
\text { sis }\end{array}$ & 13 & Y & 50 & $\begin{array}{l}\text { Chemother- } \\
\text { apy }\end{array}$ & $\begin{array}{l}\text { Favor- } \\
\text { able }\end{array}$ & NR & 2010 & 18 \\
\hline 5 & $79 y$ & M & DLBCL & Y & 7.29 & 7.4 & Y & NR & $\begin{array}{l}\text { Meth- } \\
\text { yl-prednis- } \\
\text { olone }\end{array}$ & Fatal & $<1$ & 2009 & 19 \\
\hline 6 & $71 \mathrm{y}$ & F & $\begin{array}{l}\text { NHL High } \\
\text { Grade }\end{array}$ & Y & 7.35 & 15.3 & $\mathrm{~N}$ & Normal & $\begin{array}{c}\text { Chemother- } \\
\text { apy }\end{array}$ & Fatal & $<1$ & 1996 & 20 \\
\hline 7 & $26 y$ & M & $\begin{array}{l}\text { Burkitt Lym- } \\
\text { phoma }\end{array}$ & Y & 7.23 & 7.9 & $\mathrm{~N}$ & 63 & $\begin{array}{c}\text { Chemother- } \\
\text { apy }\end{array}$ & Fatal & $<1$ & 1994 & 21 \\
\hline 8 & $74 y$ & $\mathrm{~F}$ & $\begin{array}{l}\text { T cell Lym- } \\
\text { phoma }\end{array}$ & Y & 7.34 & 14.8 & Y & 36 & $\begin{array}{l}\text { Palliative } \\
\text { care }\end{array}$ & Fatal & $<1$ & 1991 & 22 \\
\hline 9 & $71 \mathrm{y}$ & F & $\begin{array}{l}\text { Small and } \\
\text { large cell } \\
\text { Lymphoma }\end{array}$ & NR & 7.3 & 12.2 & Y & NR & $\begin{array}{c}\text { CTX, VP16, } \\
\text { BLM, VCR + } \\
\text { PDN }\end{array}$ & $\begin{array}{c}\text { Partial } \\
\text { Remis- } \\
\text { sion }\end{array}$ & 6 & 1991 & 22 \\
\hline 10 & $34 \mathrm{y}$ & F & DLBCL & NR & 7.25 & 23.6 & Y & NR & MACOB-P & $\begin{array}{l}\text { Com- } \\
\text { plete } \\
\text { Remis- } \\
\text { sion }\end{array}$ & 36 & 1991 & 22 \\
\hline 11 & $74 y$ & M & $\begin{array}{l}\text { Immature B } \\
\text { cell Lympho- } \\
\text { ma + myel- } \\
\text { odysplastic } \\
\text { syndrome }\end{array}$ & Y & 7.07 & 19.8 & NR & 47 & $\begin{array}{l}\text { Palliative } \\
\text { care }\end{array}$ & Fatal & 44 & 2010 & 23 \\
\hline
\end{tabular}




\begin{tabular}{|c|c|c|c|c|c|c|c|c|c|c|c|c|c|}
\hline 12 & $65 y$ & M & DLBCL & $\mathrm{Y}$ & 7.13 & 17 & $\mathrm{Y}$ & NR & $\begin{array}{c}\text { Chemother- } \\
\text { apy }\end{array}$ & $\begin{array}{c}\text { Favor- } \\
\text { able }\end{array}$ & NR & 2007 & 24 \\
\hline 13 & $81 \mathrm{y}$ & M & MCL & $\mathrm{Y}$ & $\begin{array}{l}\text { Lactic } \\
\text { acido- } \\
\text { sis }\end{array}$ & 13.6 & $\mathrm{~N}$ & 65 & R-CHOP & $\begin{array}{c}\text { Clinical } \\
\text { improve- } \\
\text { ment }\end{array}$ & NR & 2008 & 25 \\
\hline 14 & $79 y$ & M & AML & $\mathrm{Y}$ & $\begin{array}{l}\text { Lactic } \\
\text { acido- } \\
\text { sis }\end{array}$ & 19 & $\mathrm{~N}$ & NR & Supportive & Fatal & $<1$ & 2007 & 26 \\
\hline 15 & $75 y$ & $\mathrm{~F}$ & $\begin{array}{c}\text { Follicular } \\
\text { Lymphoma }\end{array}$ & NR & $\begin{array}{l}\text { Lactic } \\
\text { acido- } \\
\text { sis }\end{array}$ & 4.8 & $\mathrm{Y}$ & NR & $\begin{array}{c}\text { Chemother- } \\
\text { apy }\end{array}$ & Fatal & $<1$ & 2007 & 26 \\
\hline 16 & $54 \mathrm{y}$ & $\mathrm{F}$ & DLBCL & $\begin{array}{l}\text { Nor- } \\
\text { mal }\end{array}$ & $\begin{array}{l}\text { Lactic } \\
\text { acido- } \\
\text { sis }\end{array}$ & 12 & $\mathrm{Y}$ & NR & $\begin{array}{c}\text { Chemother- } \\
\text { apy }\end{array}$ & Fatal & $<1$ & 2007 & 26 \\
\hline 17 & $54 \mathrm{y}$ & M & $\begin{array}{l}\text { T cell Lym- } \\
\text { phoma }\end{array}$ & $\begin{array}{l}\text { Nor- } \\
\text { mal }\end{array}$ & $\begin{array}{l}\text { Lactic } \\
\text { acido- } \\
\text { sis }\end{array}$ & 12 & $\mathrm{~N}$ & NR & $\begin{array}{c}\text { Chemother- } \\
\text { apy }\end{array}$ & Fatal & $<3$ & 2007 & 26 \\
\hline 18 & $66 y$ & $\mathrm{~F}$ & CLL & $\begin{array}{c}\text { Nor- } \\
\text { mal }\end{array}$ & $\begin{array}{l}\text { Lactic } \\
\text { acido- } \\
\text { sis }\end{array}$ & 5.3 & $\mathrm{~N}$ & NR & $\begin{array}{c}\text { Chemother- } \\
\text { apy }\end{array}$ & Fatal & NR & 2007 & 26 \\
\hline 19 & $61 \mathrm{y}$ & $\mathrm{F}$ & Lymphoma & $\begin{array}{c}\text { Nor- } \\
\text { mal }\end{array}$ & $\begin{array}{l}\text { Lactic } \\
\text { acido- } \\
\text { sis }\end{array}$ & 11.6 & $\mathrm{~N}$ & NR & $\begin{array}{c}\text { Chemother- } \\
\text { apy }\end{array}$ & Fatal & $<1$ & 2007 & 26 \\
\hline 20 & $54 \mathrm{y}$ & M & DLBCL & $\mathrm{Y}$ & $\begin{array}{l}\text { Lactic } \\
\text { acido- } \\
\text { sis }\end{array}$ & 16.9 & $\mathrm{~N}$ & NR & $\begin{array}{c}\text { Palliative } \\
\text { care }\end{array}$ & Fatal & $<1$ & 2007 & 26 \\
\hline 21 & $74 \mathrm{y}$ & M & $\begin{array}{c}\text { Burkitt Lym- } \\
\text { phoma }\end{array}$ & $\mathrm{Y}$ & 7.29 & 15.8 & $\mathrm{~N}$ & 27 & $\begin{array}{c}\text { Palliative } \\
\text { care }\end{array}$ & Fatal & $<1$ & 2009 & 27 \\
\hline 22 & $11 \mathrm{~m}$ & $\mathrm{~F}$ & $\begin{array}{l}\text { B-cell Lym- } \\
\text { phoma }\end{array}$ & $\begin{array}{c}\text { Nor- } \\
\text { mal }\end{array}$ & $\begin{array}{l}\text { Lactic } \\
\text { acido- } \\
\text { sis }\end{array}$ & 18.6 & $\mathrm{~N}$ & NR & $\begin{array}{l}\text { B complex } \\
\text { vitamins }\end{array}$ & $\begin{array}{c}\text { Favor- } \\
\text { able }\end{array}$ & NR & 2003 & 28 \\
\hline 23 & $7 y$ & M & $\begin{array}{c}\text { pre-B cell } \\
\text { ALL }\end{array}$ & $\begin{array}{c}\text { Nor- } \\
\text { mal }\end{array}$ & 7.13 & 8.4 & $\mathrm{~N}$ & 96 & $\begin{array}{c}\text { VCR-PDN + } \\
\text { intrathecal } \\
\text { L-asparagi- } \\
\text { nase }\end{array}$ & $\mathrm{CR}$ & $>24$ & 2003 & 29 \\
\hline 24 & $29 y$ & M & T-ALL & $\begin{array}{l}\text { Nor- } \\
\text { mal }\end{array}$ & 7.29 & 11 & $\mathrm{Y}$ & NR & $\begin{array}{c}\text { Cytara- } \\
\text { bine-MTX }\end{array}$ & $\mathrm{CR}$ & & 2003 & 30 \\
\hline 25 & $64 y$ & $\mathrm{~F}$ & $\begin{array}{c}\text { DLBCL } \\
\text { extranodal }\end{array}$ & $\mathrm{Y}$ & $\begin{array}{c}\text { Lactic } \\
\text { acido- } \\
\text { sis } \\
\end{array}$ & 9.9 & $\mathrm{Y}$ & 26 & $\begin{array}{l}\text { Chemother- } \\
\text { apy }\end{array}$ & Fatal & $<1$ & 2002 & 31 \\
\hline 26 & $11 \mathrm{y}$ & $\mathrm{F}$ & T-ALL & $\mathrm{Y}$ & $\begin{array}{c}\text { Lactic } \\
\text { acido- } \\
\text { sis }\end{array}$ & 10.8 & NR & 47 & $\begin{array}{c}\text { Chemother- } \\
\text { apy }\end{array}$ & Fatal & $<1$ & 2001 & 32 \\
\hline 27 & $17 y$ & M & T-ALL & $\mathrm{N}$ & $\begin{array}{l}\text { Lactic } \\
\text { acido- } \\
\text { sis }\end{array}$ & 16 & NR & 132 & Supportive & Fatal & $<1$ & 2001 & 32 \\
\hline 28 & $18 \mathrm{y}$ & $\mathrm{F}$ & $\begin{array}{l}\text { Large-cell } \\
\text { immuno- } \\
\text { blastic T cell } \\
\text { Lymphoma }\end{array}$ & $\mathrm{Y}$ & $\begin{array}{c}\text { Lactic } \\
\text { acido- } \\
\text { sis }\end{array}$ & 15.4 & NR & 44 & $\begin{array}{c}\text { Chemother- } \\
\text { apy }\end{array}$ & Fatal & $<1$ & 2001 & 32 \\
\hline 29 & $82 y$ & M & $\begin{array}{l}\text { Hodgkin's } \\
\text { Disease }\end{array}$ & $\mathrm{Y}$ & 7.24 & 11.5 & $\mathrm{~N}$ & NR & $\begin{array}{l}\text { Chemother- } \\
\text { apy }\end{array}$ & Fatal & $<1$ & 2001 & 33 \\
\hline 30 & $28 y$ & M & $\begin{array}{l}\text { NK/T-Cell } \\
\text { Lymphoma }\end{array}$ & $\mathrm{Y}$ & 7.17 & 11.2 & $\mathrm{Y}$ & 42 & $\begin{array}{c}\text { Chemother- } \\
\text { apy }\end{array}$ & Fatal & $<2$ & 2007 & 34 \\
\hline 31 & $64 y$ & M & SCLC & NR & 7.18 & 15.8 & $\mathrm{Y}$ & NR & $\begin{array}{c}\text { Dialysis for } \\
\text { acidosis }\end{array}$ & Fatal & $<1$ & 2006 & 35 \\
\hline
\end{tabular}




\begin{tabular}{|c|c|c|c|c|c|c|c|c|c|c|c|c|c|}
\hline 32 & $24 \mathrm{y}$ & M & $\begin{array}{l}\text { B-cell im- } \\
\text { munoblastic } \\
\text { lymphoma } \\
\text { with AML M3 } \\
\text { transforma- } \\
\text { tion }\end{array}$ & $\mathrm{Y}$ & 7.05 & 12 & $\begin{array}{l}\text { Y (in- } \\
\text { creased } \\
\text { liver en- } \\
\text { zymes) }\end{array}$ & 38 & $\begin{array}{l}\text { Dialysis for } \\
\text { acidosis } \\
\text { and chemo- } \\
\text { therapy }\end{array}$ & Fatal & $<1$ & 2005 & 36 \\
\hline 33 & $77 y$ & M & $\begin{array}{l}\text { MCL blastoid } \\
\text { variant }\end{array}$ & NR & 7.27 & 26.3 & $\mathrm{~N}$ & NR & $\begin{array}{l}\text { Chemother- } \\
\text { apy }\end{array}$ & CR & & 2004 & 37 \\
\hline 34 & $7 y$ & M & $\begin{array}{c}\text { pre-B- cell } \\
\text { ALL }\end{array}$ & $\mathrm{Y}$ & 7.09 & 5.5 & $\mathrm{~N}$ & $<45$ & $\begin{array}{l}\text { Palliative } \\
\text { care }\end{array}$ & Fatal & $<1$ & 2009 & 38 \\
\hline 35 & $33 y$ & M & $\begin{array}{l}\text { Anaplastic } \\
\text { Large B-cell } \\
\text { Lymphoma }\end{array}$ & NR & 7.06 & 11.1 & $\mathrm{Y}$ & NR & NR & Fatal & $<1$ & 2008 & 39 \\
\hline 36 & $48 y$ & $\mathrm{~F}$ & $\begin{array}{l}\text { Anaplastic } \\
\text { Large B-cell } \\
\text { Lymphoma }\end{array}$ & NR & $\begin{array}{l}\text { Lactic } \\
\text { acido- } \\
\text { sis }\end{array}$ & 6.19 & Y & NR & NR & Fatal & $<1$ & 2008 & 39 \\
\hline 37 & $45 y$ & $\mathrm{~F}$ & $\begin{array}{l}\text { Anaplastic } \\
\text { Large B-cell } \\
\text { Lymphoma }\end{array}$ & NR & $\begin{array}{l}\text { Lactic } \\
\text { acido- } \\
\text { sis } \\
\end{array}$ & 6.5 & $\mathrm{Y}$ & NR & NR & Fatal & $<1$ & 2008 & 39 \\
\hline 38 & $14 \mathrm{y}$ & $\mathrm{F}$ & $\begin{array}{c}\text { Cancer of } \\
\text { unknown } \\
\text { primary site }\end{array}$ & NR & $\begin{array}{l}\text { Lactic } \\
\text { acido- } \\
\text { sis }\end{array}$ & 22.08 & $\mathrm{Y}$ & NR & NR & Fatal & $<1$ & 2004 & 40 \\
\hline 39 & $25 y$ & $\mathrm{~F}$ & $\begin{array}{c}\text { Cancer of } \\
\text { unknown } \\
\text { primary site }\end{array}$ & $\begin{array}{l}\text { Nor- } \\
\text { mal }\end{array}$ & 7.08 & 19.03 & $\mathrm{Y}$ & 91 & $\begin{array}{l}\text { Dialysis for } \\
\text { acidosis }\end{array}$ & Fatal & $<1$ & 2002 & 41 \\
\hline 40 & $70 y$ & M & $\begin{array}{l}\text { Cholangio- } \\
\text { carcinoma }\end{array}$ & $\begin{array}{l}\text { Nor- } \\
\text { mal }\end{array}$ & $\begin{array}{l}\text { Lactic } \\
\text { acido- } \\
\text { sis }\end{array}$ & $12: 05$ & $\mathrm{Y}$ & NR & $\begin{array}{l}\text { Dialysis for } \\
\text { acidosis }\end{array}$ & Fatal & $<1$ & 2011 & 42 \\
\hline 41 & $55 \mathrm{y}$ & M & $\begin{array}{l}\text { Multiple } \\
\text { Myeloma }\end{array}$ & $\begin{array}{l}\text { Nor- } \\
\text { mal }\end{array}$ & $\begin{array}{l}\text { Lactic } \\
\text { acido- } \\
\text { sis }\end{array}$ & 6.3 & NR & Normal & $\begin{array}{l}\text { Chemother- } \\
\text { apy }\end{array}$ & Fatal & $<1$ & 2002 & 43 \\
\hline 42 & $81 \mathrm{y}$ & M & DLBCL & $\mathrm{Y}$ & 7.29 & 13.4 & $\mathrm{~N}$ & 37 & $\begin{array}{l}\text { Palliative } \\
\text { care }\end{array}$ & Fatal & $<1$ & 2013 & 1 \\
\hline 43 & $73 y$ & M & $\begin{array}{l}\text { CLL with } \\
\text { DLBCL trans- } \\
\text { formation }\end{array}$ & $\mathrm{Y}$ & $\begin{array}{l}\text { Lactic } \\
\text { acido- } \\
\text { sis } \\
\end{array}$ & 13 & $\mathrm{~N}$ & $<60$ & $\begin{array}{l}\text { Palliative } \\
\text { care }\end{array}$ & Fatal & $<1$ & 2016 & 44 \\
\hline 44 & $58 y$ & M & ALL & $\begin{array}{c}\text { Nor- } \\
\text { mal }\end{array}$ & 7.28 & 13.5 & $\mathrm{Y}$ & Normal & $\begin{array}{l}\text { Chemother- } \\
\text { apy }\end{array}$ & $\mathrm{CR}$ & & 2017 & 10 \\
\hline 45 & $51 \mathrm{y}$ & $\mathrm{F}$ & DLBCL & NR & 7.18 & 18.3 & $\mathrm{Y}$ & NR & $\begin{array}{l}\text { Chemother- } \\
\text { apy }\end{array}$ & $\begin{array}{l}\text { Clinical } \\
\text { improve- } \\
\text { ment }\end{array}$ & NR & 2013 & 45 \\
\hline 46 & $66 y$ & $\mathrm{~F}$ & $\begin{array}{c}\text { NK/T-Cell } \\
\text { Lymphoma }\end{array}$ & $\mathrm{Y}$ & 7.23 & 24 & $\mathrm{~N}$ & 11 & $\begin{array}{l}\text { Chemother- } \\
\text { apy }\end{array}$ & Fatal & $<1$ & 2016 & 14 \\
\hline 47 & $67 y$ & M & MCL & $\begin{array}{l}\text { Nor- } \\
\text { mal }\end{array}$ & 7.23 & 11.3 & $\mathrm{Y}$ & 91 & $\begin{array}{l}\text { Chemother- } \\
\text { apy }\end{array}$ & $\mathrm{CR}$ & & 2018 & 46 \\
\hline 48 & $42 \mathrm{y}$ & M & AML & $\mathrm{Y}$ & 7.12 & 19.75 & $\mathrm{~N}$ & 54 & $\begin{array}{l}\text { Palliative } \\
\text { care }\end{array}$ & Fatal & $<1$ & 1985 & 47 \\
\hline 49 & $71 \mathrm{y}$ & M & $\begin{array}{l}\text { intravascular } \\
\text { large B-cell } \\
\text { Lymphoma }\end{array}$ & $\mathrm{Y}$ & $\begin{array}{l}\text { Lactic } \\
\text { acido- } \\
\text { sis }\end{array}$ & 8.8 & $\begin{array}{l}\text { Y (in- } \\
\text { creased } \\
\text { liver en- } \\
\text { zymes) }\end{array}$ & 26 & $\begin{array}{l}\text { Chemother- } \\
\text { apy }\end{array}$ & $\begin{array}{l}\text { Favor- } \\
\text { able }\end{array}$ & NR & 2012 & 48 \\
\hline 50 & $57 y$ & M & $\begin{array}{l}\text { AML (M4 } \\
\text { type) }\end{array}$ & $\mathrm{Y}$ & 7.35 & 14 & $\mathrm{~N}$ & NR & $\begin{array}{l}\text { Chemother- } \\
\text { apy }\end{array}$ & PR & $\begin{array}{l}<2 \text { (for } \\
\text { recur- } \\
\text { rence) }\end{array}$ & 2018 & 9 \\
\hline 51 & $74 y$ & $\mathrm{~F}$ & Lymphoma & $\mathrm{Y}$ & 7.23 & 20 & $\mathrm{Y}$ & 50 & $\begin{array}{l}\text { Palliative } \\
\text { care }\end{array}$ & Fatal & $<1$ & 2019 & 49 \\
\hline
\end{tabular}




\begin{tabular}{|c|c|c|c|c|c|c|c|c|c|c|c|c|c|}
\hline 52 & $45 \mathrm{y}$ & $\mathrm{F}$ & $\begin{array}{c}\text { Cervical } \\
\text { squamous } \\
\text { cell carcino- } \\
\text { ma }\end{array}$ & Y & 7.23 & 9.2 & $\mathrm{~N}$ & 18 & $\begin{array}{l}\text { Palliative } \\
\text { care }\end{array}$ & Fatal & $<1$ & 2018 & 50 \\
\hline 53 & $73 y$ & M & $\begin{array}{c}\text { Lym- } \\
\text { pho-plas- } \\
\text { macytic } \\
\text { lymphoma } \\
\text { with DLBCL } \\
\text { transforma- } \\
\text { tion }\end{array}$ & $\mathrm{Y}$ & 7.31 & 29 & $\mathrm{~N}$ & 51 & $\begin{array}{l}\text { Palliative } \\
\text { care }\end{array}$ & Fatal & $<1$ & 2013 & 51 \\
\hline
\end{tabular}

Legend: NR - Not Reported; CR - Complete Remission; PR - Partial Remission; DLBCL - Diffuse Large B-Cell Lymphoma; NHL - Non-Hodgkin's Lymphoma; MCL - Mantle-Cell Lymphoma; AML - Acute Myeloid Leukemia; ALL - Acute Lymphoblastic Leukemia; CLL - Chronic Lymphocytic Leukaemia.

A literary review of 53 relevant clinical cases published between the years 1985-2019, focused on cases of haematological malignancies associated with lactic acidosis, has shown that as a consequence of the increased glucose consumption by the tumour, $29(54.7 \%)$ of these patients were in a state of persistent hypoglycaemia and in 17 cases (32.1\%) there was no report of neurologic deficiencies. This peculiarity is due to an ability of the brain to employ lactate as a primary source of energy, providing the brain with a protective mechanism from systemic hypoglycaemia. However, the mechanism by which this phenomenon occurs remains undetermined [11-13]. Among the 53 reviewed cases, 26 cases reported liver involvement in the development of the pathology, out of which $69.2 \%$ of the cases resulted in fatalities. It is relevant to note that the other $30.8 \%$ of cases (with reported favourable outcomes) have all engaged in a protocol of chemotherapy. The unusual clinical presentation that we are reporting, characterised by severe, untreatable hypoglycaemia, lactic acidosis, and liver involvement, was only described in 11 malignancy cases, all are cases of lymphoma, and predicts a very poor prognosis (Table 3 ).

Presumably, the poor prognoses are due to the aggressive nature of the disease, the fact that these cases are often diagnosed at a rather late stage in the disease progression, and indeed, any part of the clinical presentation may be justifiably suspected as a more prevalent condition. However, it is precisely because of the rarity and peculiarity of this clinical presentation that we believe the consideration and exclusion of a potential hematological emergency should be prioritised in the clinical approach. The Warburg effect, stemming from the alteration of cancer metabolism, seem to be manipulable using dichloroacetate (DCA), which is not yet indicated for the treatment of cancer but is used to treat lactic acidosis and diabetes. Despite the lack of official indication for DCA in this setting, there is an accumulating body of evidence to support its efficacy. DCA is thought to mitigate the Warburg effect by inhibiting pyruvate dehydrogenase kinase, leading to the activation of pyruvate dehydrogenase, and by that process DCA is able to stimulate cellular respiration through oxidative phosphorylation, rather than strict glycolysis, offsetting the relative metabolic advantages that neoplastic tissues hold over their surrounding healthy tissue. Moreover, due to the activation of mitochondrial respiration, DCA can limit the amount of lactate produced by the tumour, changing its chemical environment and inhibiting its cellular proliferation, as well as restoring a degree of apoptotic function through the increase in cytochrome c [52].

However, although DCA represents a promising prospect, it is important to note that the encouragement of mitochondrial activity could have deleterious consequences for the nervous system, as these tissues rely primarily on glycolysis and may lack the cellular functions to sustain the increase in free radicals. Nevertheless, in the context of Warburgism, where the severe systemic hypoglycaemia does not result in neuroglycopenic symptoms, it seems reasonable to hypothesise that the cells of the nervous system have already gone through the necessary remodeling to endure this metabolic shift. However, we are currently unaware of such research.

\section{References}

1. Tanios G, Aranguren IM, Goldstein JS, Patel CB (2013) Diffuse large B-cell lymphoma: A metabolic disorder? The American Journal of Case Reports 14: 518-525.

2. Spinazzé S, Schrijvers D (2006) Metabolic emergencies. Critical reviews in oncology/hematology 58(1): 79-89.

3. Luft D, Deichsel G, Schmulling RM, Stein W, Eggstein M (2019) Definition of clinically relevant lactic acidosis in patients with internal diseases. American Journal of Clinical Pathology 80(4): 484-489.

4. Vanhove K, Graulus GJ, Mesotten L (2019) The metabolic landscape of lung cancer: new insights in a disturbed glucose metabolism. Frontiers in Oncology 9: 1215.

5. Dalton SD, Rahimi AR (2001) Emerging role of riboflavin in the treatment of nucleoside analogue-induced type B lactic acidosis. Aids Patient Care and Stds 15(12): 611-614.

6. Shaer AJ, Rastegar A (2000) Lactic acidosis in the setting of antiretroviral therapy for the acquired immunodeficiency syndrome. American Journal of Nephrology 20(4): 332-338.

7. Warburg $O$ (1956) On the origin of cancer cells. Science 123(3191): 309-314.

8. Mazurek S, Boschek CB, Eigenbrodt E (1997) The role of phosphometabolites in cell proliferation, energy metabolism, and 


\section{Juniper Online Journal of Case Studies}

tumor therapy. Journal of Bioenergetics and Biomembranes 29(4): 315-330.

9. Brault C, Zerbib Y, Delette C (2018) The Warburg effect as a type B lactic acidosis in a patient with acute myeloid leukemia: a diagnostic challenge for clinicians. Frontiers in Oncology 8: 232.

10. Sayyed AH, Aleem A, Al-Katari MS (2018) Acute lymphoblastic leukemia presenting with liver infiltration and severe lactic acidosis. The American Journal of Case Reports 19: 453-457.

11. King P, Parkin H, Macdonald IA, Barber C, Tattersall RB (1997) The effect of intravenous lactate on cerebral function during hypoglycaemia. Diabetic Medicine 14(1): 19-28.

12. Veneman T, Mitrakou A, Mokan M, Cryer P, Gerich J (1994) Effect of hyperketonemia and hyperlacticacidemia on symptoms, cognitive dysfunction, and counterregulatory hormone responses during hypoglycemia in normal humans. Diabetes 43(11): 1311-1317.

13. Smith D, Pernet A, Hallett WA, Bingham E, Marsden PK, et al. (2003) Lactate: a preferred fuel for human brain metabolism in vivo. Journal of Cerebral Blood Flow \& Metabolism 23(6): 658-664.

14. Henkle B, Arndt P (2017) A 66-year-old woman with sudden onset of disseminated intravascular coagulation, lactic acidosis, and hypoglycemia. Chest 151(2): e41-e44.

15. Elhomsy GC, Eranki V, Albert SG, Fesler MJ, Parker SM, et al. (2012) "Hyper-Warburgism", a cause of asymptomatic hypoglycemia with lactic acidosis in a patient with non-Hodgkin's lymphoma. The Journal of Clinical Endocrinology \& Metabolism 97(12): 4311-4316.

16. Ruiz JP, Singh A, Hart P (2011) Type B lactic acidosis secondary to malignancy: case report, review of published cases, insights into pathogenesis, and prospects for therapy. Scientific World Journal 7(11): 1316-1324.

17. Huo L, Luo Y, Zhang T, Zhu Z, Li F (2010) Unexpected primary osseous lymphoma as the cause of lactic acidosis in a patient suffering from pancreatitis. Clinical nuclear medicine 35(10): 790-793.

18. Keller BC, Nussensveig D, Dowell JE (2010) Diffuse large B-cell lymphoma in a hepatitis $C$ virus-infected patient presenting with lactic acidosis and hypoglycemia. The American Journal of the Medical Sciences 339(2): 202-204.

19. Jung B, Zoric L, Chanques G, Konate A, Nocca D, et al. (2010) Acute abdomen and severe lactic acidosis can lead to a surprising diagnosis. Intensive Care Medicine 36(1): 169-170.

20. Dürig J, Fiedler W, De Wit M, Steffen M, Hossfeld DK (1996) Lactic acidosis and hypoglycemia in a patient with high-grade non-Hodgkin's lymphoma and elevated circulating TNF- $\alpha$. Annals of hematology 72(2): 97-99.

21. Bergin C, Pilkington R, Mc Creary C, Mulcahy F, Crowley V (1994) Lactic acidosis non-Hodgkin lymphoma and the acquired immunodeficiency syndrome. Genitourinary Medicine 70(2): 148-149.

22. Caspar CB, Oelz O (1991) Lactic acidosis in malignant lymphoma. The American Journal of Medicine 91(2): 197-198.

23. Diaz J, Antoine J, Azad N (2010) A case of hypoglycemia, lactic acidosis, and hematologic malignancy. Endocrine Practice 16(2): 241-243.

24. Prikis M, Bhasin V, Young MP, Gennari FJ, Rimmer JM (2007) Sustained low-efficiency dialysis as a treatment modality in a patient with lymphoma-associated lactic acidosis. Nephrology Dialysis Transplantation 22(8): 2383-2385

25. Diab K, Knox KS, Hage CA (2008) An 81-year-old man with lactic acidosis, refractory hypoglycemia, and lymphocytosis. Chest 133(1)
306-310.

26. Friedenberg AS, Brandoff DE, Schiffman FJ (2007) Type B lactic acidosis as a severe metabolic complication in lymphoma and leukemia: a case series from a single institution and literature review. Medicine 86(4): 225-232.

27. Glasheen J, Sorensen M (2005) Burkitt's lymphoma presenting with lactic acidosis and hypoglycemia-a case presentation. Leukemia \& Lymphoma 46(2): 281-283.

28. Svahn J, Schiaffino MC, Caruso U, Calvillo M, Minniti G, et al. (2003) Severe lactic acidosis due to thiamine deficiency in a patient with B-cell leukemia/lymphoma on total parenteral nutrition during high-dose methotrexate therapy. Journal of Pediatric Hematology/ Oncology 25(12): 965-968.

29. Hayek M, Srinivasan A (2003) Acute lymphoblastic leukemia presenting with lactic acidosis and renal tubular dysfunction. Journal of Pediatric Hematology/Oncology 25(6): 488-490.

30. De Keulenaer B, Van Outryve S, De Backer A, Overbeke LV, Daelemans $\mathrm{R}$, et al. (2003) Symptomatic lactic acidosis due to relapse of T-cell acute lymphoblastic leukaemia in the kidney. Nephrology Dialysis Transplantation 18(6): 1214-1216.

31. Di Comite G, Dagna L, Piatti PM, Monti LD, Tantardini F, et al. (2002) Hypoglycaemia and lactic acidosis in a MALT non Hodgkin's lymphoma. Leukemia \& Lymphoma 43(6): 1341-1342.

32. Sillos EM, Shenep JL, Burghen GA, Pui CH, Behm FG, et al. (2001) Lactic acidosis: a metabolic complication of hematologic malignancies: case report and review of the literature. Cancer 92(9): 2237-2246.

33. Thakur V, Sander G, Rab ST (2001) Hodgkin's disease and lactic acidosis. Nephron 88(3): 276-277.

34. He YF, Wei W, Sun ZM, Ji CS, Wang G, et al. (2007) Fatal lactic acidosis and hypoglycemia in a patient with relapsed natural killer/T-cell lymphoma. Advances in Therapy 24(3): 505-509.

35. Manuel B, Suresh V, Saphwat E (2006) Refractory metabolic acidosis in small cell cancer of the lung. Southern Medical Journal 99(7): 782-784?

36. Dogan E, Erkoc R, Sayarlioglu H, Alici S, Dilek I, et al. (2005) Fatal lactic acidosis due to leukemic transformation in a patient with nonHodgkin's lymphoma: case report. Advances in Therapy 22(5): 443446.

37. Ohtsubo K, Imamura R, Seki R, Ohshima K, Hashiguchi M, et al. (2004) Blastoid variant of mantle cell lymphoma with lactic acidosis: a case report. International Journal of Hematology 80(5): 428-431.

38. Luscri N, Mauer M, Sarafoglou K, Moran A, Tolar J (2009) Lactic acidosis and hypoglycemia with ALL relapse following engrafted bone marrow transplant. Pediatric Blood \& Cancer 53(2): 223-225.

39. Mosunjac M, Sundstrom B, Mosunjac MI (2008) Unusual presentation of anaplastic large cell lymphoma with clinical course mimicking fever of unknown origin and sepsis: autopsy study of five cases. Croatian Medical Journal 49(5): 660-668.

40. Cheng JC, Esparza SD, Knez VM, Sakamoto KM, Moore TB (2004) Severe lactic acidosis in a 14-year-old female with metastatic undifferentiated carcinoma of unknown primary. Journal of Pediatric Hematology/ Oncology 26(11): 780-782.

41. Chau WK, Yang CF, Chou Y, Ho CH (2002) Aggressive undifferentiated carcinoma of unknown primary site complicated by lactic acidosis after bleeding: a case report. Japanese Journal of Clinical Oncology 32(6): 210-214 
42. Wall BM, Mansour N, Cooke CR (2000) Acute fulminant lactic acidosis complicating metastatic cholangiocarcinoma. The American Journal of the Medical Sciences 319(2): 126-129.

43. Ustun C, Fall P, Szerlip HM (2002) Multiple myeloma associated with lactic acidosis. Leukemia \& Lymphoma 43(12): 2395-2397.

44. Soleja M, Mims M, Rivero G (2016) Uncovering molecular abnormalities leading to the Warburg effect in primary refractory diffuse large B-cell lymphoma. Blood Cancer Journal 6(12): e502-e502.

45. Kuo CY, Yeh ST, Huang CT, Lin SF (2014) Diffuse large B-cell lymphoma presenting with type B lactic acidosis and hemophagocytic syndrome. The Kaohsiung Journal of Medical Sciences 30(8): 428-429.

46. Wahab A, Kesari KJ, Smith S, Liu Y, Barta SK (2018) Type B lactic acidosis, an uncommon paraneoplastic syndrome. Cancer Biology \& Therapy 19(2): 101-104.

47. Makino H, Noda K, Inagaki Y, Horie H, Osegawa M, et al. (1985) Lactic acidosis and hypoglycemia associated with acute leukemia. Japanese Journal of Medicine 24(3): 257-262.

48. Kloesel B, Vaidya R, Howard MT (2013) A unifying diagnosis for pancytopenia, fever, hypoglycemia, and lactic acidosis. American Journal of Hematology 88(1): 78-81.

49. Talwalkar PG (2019) Severe and persistent hypoglycemia with lactic acidosis in an elderly lady with type 2 diabetes mellitus and lymphoma leukemia: A rare case report. Diabetes \& Metabolic Syndrome: Clinical Research \& Reviews 13(1): 648-650.

50. Hausken J, Haave EM, Haugaa H, Loberg EM, Kongsgaard UE (2019) A patient with solid gynecologic cancer causing lactic acidosis, severe hypercalcemia, and hypoglycemia. Clinical Case Reports 7(1): 64-70.

51. Buppajarntham S, Junpaparp P, Kue A Pai P (2013) Warburg effect associated with transformed lymphoplasmacytic lymphoma to diffuse large B-cell lymphoma. The American Journal of Emergency Medicine 31(6): 999-e5-1006-e5.

52. Tataranni T, Piccoli C (2019) Dichloroacetate (DCA) and Cancer: an overview towards clinical applications. Oxid Med Cell Longev.

\section{Your next submission with Juniper Publishers} will reach you the below assets

- Quality Editorial service

- Swift Peer Review

- Reprints availability

- E-prints Service

- Manuscript Podcast for convenient understanding

- Global attainment for your research

- Manuscript accessibility in different formats

( Pdf, E-pub, Full Text, Audio)

- Unceasing customer service

Track the below URL for one-step submission

https://juniperpublishers.com/online-submission.php 